\title{
Proteomic characterisation reveals active Wnt-signalling by human multipotent stromal cells as a key regulator of beta cell survival and proliferation
}

\author{
Miljan Kuljanin $^{1}$ • Gillian I. Bell ${ }^{2}$ Stephen E. Sherman ${ }^{2,3}$ • Gilles A. Lajoie $^{1}$ • \\ David A. Hess ${ }^{2,3}$
}

Received: 23 February 2017 / Accepted: 23 May 2017 /Published online: 14 July 2017

(C) Springer-Verlag GmbH Germany 2017

\begin{abstract}
Aims/hypothesis Novel strategies to stimulate the expansion of beta cell mass in situ are warranted for diabetes therapy. The aim of this study was to elucidate the secretome of human bone marrow (BM)-derived multipotent stromal cells (MSCs) with documented islet regenerative paracrine function. We hypothesised that regenerative MSCs will secrete a unique combination of protein factors that augment islet regeneration.

Methods Human BM-derived MSCs were examined for glucose-lowering capacity after transplantation into streptozotocin-treated NOD/severe combined immunodeficiency (SCID) mice and segregated into samples with regenerative $\left(\mathrm{MSC}^{\mathrm{R}}\right)$ vs nonregenerative $\left(\mathrm{MSC}^{\mathrm{NR}}\right)$ capacity. Secreted proteins associated with islet regenerative function were identified using stable isotope labelling with amino acids in cell culture (SILAC)-based quantitative proteomics. To functionally validate the importance of active Wnt signalling,
\end{abstract}

Electronic supplementary material The online version of this article (doi:10.1007/s00125-017-4355-7) contains peer-reviewed but unedited supplementary material, which is available to authorised users.

David A. Hess

dhess@robarts.ca

$\triangle$ Gilles A. Lajoie

glajoie@uwo.ca

1 Don Rix Protein Identification Facility, Department of Biochemistry, Schulich School of Medicine and Dentistry, Western University, London, ON N5A 6C1, Canada

2 Department of Physiology and Pharmacology, Schulich School of Medicine and Dentistry, Western University, London, ON, Canada

3 Molecular Medicine Laboratories, Krembil Centre for Stem Cell Biology, Robarts Research Institute, 100 Perth Drive,

London, ON N6A 5K8, Canada we stimulated the Wnt-signalling pathway in $\mathrm{MSC}^{\mathrm{NR}}$ samples during ex vivo expansion using glycogen synthase kinase 3 (GSK3) inhibition (CHIR99201), and the conditioned culture media $(\mathrm{CM})$ generated was tested for the capacity to support cultured human islet cell survival and proliferation in vitro. Results $\mathrm{MSC}^{\mathrm{R}}$ showed increased secretion of proteins associated with cell growth, matrix remodelling, immunosuppressive and proangiogenic properties. In contrast, $\mathrm{MSC}^{\mathrm{NR}}$ uniquely secreted proteins known to promote inflammation and negatively regulate angiogenesis. Most notably, $\mathrm{MSC}^{\mathrm{R}}$ maintained Wnt signalling via Wnt5A/B ( 2.5 -fold increase $)$ autocrine activity during ex vivo culture, while $\mathrm{MSC}^{\mathrm{NR}}$ repressed Wnt signalling via Dickkopf-related protein (DKK)1 ( 2.5-fold increase) and DKK3 secretion. Inhibition of GSK3 activity in $\mathrm{MSC}^{\mathrm{NR}}$ samples increased the accumulation of nuclear $\beta$-catenin and generated CM that augmented beta cell survival (13\% increases) and proliferation when exposed to cultured human islets.

Conclusions/interpretation Maintenance of active Wnt signalling within human MSCs promotes the secretion of matricellular and proangiogenic proteins that formulate a niche for islet regeneration.

Keywords Angiogenesis · Diabetes · Inflammation · Islets · Mass spectrometry $\cdot$ Multipotent stromal cells $\cdot$ Proteomics · Wnt signalling

$\begin{array}{ll}\text { Abbreviations } \\ \text { 7AAD } & \text { 7-Aminoacetinomycin D } \\ \text { ADAMTS } & \begin{array}{l}\text { A disintegrin and metalloproteinase with } \\ \text { thrombospondin motifs }\end{array} \\ \text { BM } & \begin{array}{l}\text { Bone marrow } \\ \text { BMP }\end{array} \\ \text { CM } & \text { Bone morphogenetic protein } \\ \text { Conditioned culture media }\end{array}$




$\begin{array}{ll}\text { DKK } & \text { Dickkopf-related protein } \\ \text { EBM } & \text { Endothelial basal media } \\ \text { ECM } & \text { Extracellular matrix } \\ \text { EdU } & \text { 5-Ethynyl-2'-deoxyuridine } \\ \text { EGM } & \text { Endothelial growth medium } \\ \text { FGF } & \text { Fibroblast growth factor } \\ \text { FGFR } & \text { Fibroblast growth factor receptor } \\ \text { FLT1/4 } & \text { Vascular endothelial growth factor receptor 1/4 } \\ \text { Flz3 } & \text { FluoZin-3 } \\ \text { FZD } & \text { Frizzled } \\ \text { GSK3 } & \text { Glycogen synthase kinase 3 } \\ \text { HMVEC } & \text { Human microvascular endothelial cells } \\ \text { IIDP } & \text { Integrated Islet Distribution Program } \\ \text { KDR } & \text { Vascular endothelial growth factor receptor 2 } \\ \text { LFQ } & \text { Label-free quantification } \\ \text { MMP } & \text { Matrix metalloproteinase } \\ \text { MSC } & \text { Multipotent stromal cell } \\ \text { MSC } & \text { Nonregenerative MSC } \\ \text { MSC } & \text { Regenerative MSC } \\ \text { PDGF } & \text { Platelet-derived growth factor } \\ \text { SCID } & \text { Severe combined immunodeficiency } \\ \text { SFRP1 } & \text { Secreted frizzled-related protein 1 } \\ \text { SILAC } & \text { Stable isotope labelling with amino acids in cell } \\ & \text { culture } \\ \text { STZ } & \text { Streptozotocin } \\ \text { TIMP } & \text { Metalloproteinase inhibitor } \\ \text { VEGF } & \text { Vascular endothelial growth factor receptor } \\ \text { WISP2 } & \text { Wnt-inducible signalling pathway protein 2 } \\ & \end{array}$

\section{Introduction}

In 2013, WHO reported that over 347 million people worldwide lived with diabetes, and estimated that this number will increase to 552 million by 2030, making diabetes a worldwide epidemic [1]. The Edmonton Protocol provided proof-ofconcept that islet transplantation can temporarily reduce insulin dependence in individuals with type 1 diabetes [2]. Although islet replacement is an attractive strategy to combat diabetes, a critical shortage of donor islets and eventual rejection by continuing autoimmunity prevent the widespread application of this approach [3]. Bone marrow (BM)-derived stem cell transplantation has also been reported to promote endogenous islet regeneration in preclinical models, and represents a promising alternative strategy to combat diabetes [4].

Human multipotent stromal cells (MSCs) have become a focal point in recent clinical trials for tissue repair [5]. MSCs are readily available from autologous or allogenic donors, they are efficiently expanded in culture and they home to damaged tissues to initiate innate repair mechanisms [6]. MSCs exert their therapeutic effects primarily through the secretion of trophic signals within damaged organs, or impact tissue repair from distant sites by secretion of regenerative effectors into the circulation within exosomes or microvesicles [7]. Exosomes harvested from MSCs have been shown to contain proangiogenic proteins and promote the healing of ischaemic tissue [8,9]. MSCs also secrete a wide variety of immunomodulatory molecules that dampen autoimmunity via modulation of immune cell functions $[10,11]$. Currently, the mechanisms underlying the regenerative and immunomodulatory effects of MSCs remain poorly understood, and better understanding of MSC protein secretion is required to harness the true regenerative capacity of MSCs.

In the context of diabetes, transplanted human BM-derived MSCs have been shown to promote repair of pancreatic islets and renal glomeruli in NOD/severe combined immunodeficiency (SCID) mice after beta cell ablation [6]. Although MSC conversion into insulin-expressing beta-like cells has been demonstrated after stable induction of PDX1 [12], minimally manipulated MSCs did not adopt a beta cell phenotype after transplantation in vivo. In contrast, transplanted MSCs initiated endogenous islet recovery via paracrine stimulation [13]. In a series of publications, we have shown that human BM-derived MSCs stimulated the emergence of small, recipient-derived islet-like structures associated with the ductal epithelial niche within 7 days of injection into streptozotocin (STZ)-treated hyperglycaemic NOD/SCID mice $[14,15]$. Unfortunately, MSC samples showed donordependent variability in the capacity to improve glycaemia and prolonged expansion ex vivo reduced islet regenerative prowess [14]. Thus, detailed proteomic analyses of the islet regenerative MSC secretome remain the key to understanding which protein signals promote islet regeneration in situ.

We compared the secretory protein profile of human BMderived MSCs with or without islet regenerative capacity by performing global proteomic analysis of conditioned culture media $(\mathrm{CM})$ after stable isotope labelling with amino acids in cell culture (SILAC) [16]. We hypothesised that islet regenerative MSCs will secrete a combination of unique protein factors that augment islet regeneration. In order to confirm our findings we also examined the effects of exogenous stimulation of Wnt signalling in MSCs for the survival and proliferation of human islet-derived beta cells in vitro. To our knowledge, this is the first proteomic study linking the human MSC secretome profile with beta cell regenerative function and it highlights the importance of active Wnt signalling in the maintenance of MSC-induced islet regeneration.

\section{Methods}

$M_{S C}{ }^{R}$ reduced hyperglycaemia after transplantation in vivo Human $B M$ was obtained from healthy donors after informed consent at the London Health Sciences Centre (London, ON, Canada). All studies were approved by the human ethics committee at Western University (2015-003). 
The hyperglycaemia-lowering capacity of six MSC samples was assessed after tail vein injection of 500,000 cells into STZ-treated NOD/SCID mice as previously described [14]. Blood glucose concentrations were monitored weekly for 42 days and samples were segregated into regenerative $\left(\mathrm{MSC}^{\mathrm{R}}\right.$ ) or nonregenerative (MSC ${ }^{\mathrm{NR}}$ ) based on the ability to reduce blood glucose compared with PBS-injected control mice.

MSC culture and SILAC labelling MSCs were expanded in AmnioMax media (Invitrogen Carlsbad, CA, USA). At passage 3 MSCs were switched to custom AmnioMax that contained no L-arginine or L-lysine. Heavy $\left[{ }^{13} \mathrm{C}_{6}{ }^{15} \mathrm{~N}_{4}\right]$ L-arginine and $\left[{ }^{13} \mathrm{C}_{6}{ }^{15} \mathrm{~N}_{2}\right]$ L-lysine were added into SILAC media at $87.8 \mathrm{mg} / 1$ and $52.2 \mathrm{mg} / 1$, respectively (Silantes Germany). Excess unlabelled L-proline was added to prevent conversion of heavy arginine into heavy proline [17]. MSCs were grown in SILAC media for 9 days to achieve $>95 \%$ label incorporation.

Generation of labelled CM and proteomic workflows MSCs were re-plated in AmnioMax without supplement for $24 \mathrm{~h}$ to collect proteins secreted by MSCs. CM was collected in biological and technical duplicate, filtered and concentrated using $3 \mathrm{kDa}$ cut-off filter units (Millipore, Bedford, MA, USA). Total protein $(150 \mu \mathrm{g})$ was subjected to 1D SDSPAGE fractionation using $12 \%$ gels and quantified using the bicinchoninic acid assay (BCA). All MS data was collected using a QExactive (ThermoFisher Scientific, Waltham, MA, USA). Full MS parameters are outlined in the electronic supplementary material (ESM) Table 1. Data analysis was performed using PeaksStudio 7.5 and MaxQuant 1.5.2.8. See ESM Methods for complete details.

HMVEC tubule forming assay To assess CM influence on endothelial cell function in vitro, 120,000 human microvascular endothelial cells (HMVECs) were cultured on growth factorreduced Geltrex (Life Technologies, Carlsbad, CA, USA) in endothelial basal media (EBM-2; Lonza, NJ, USA) conditioned by $\mathrm{MSC}^{\mathrm{R}}$ and $\mathrm{MSC}^{\mathrm{NR}}$ for $24 \mathrm{~h}$. As a positive control, HMVECs were also grown in Geltrex bathed in complete endothelial growth medium (EGM-2 = EBM-2 + 5\% FBS + IGF, basic fibroblast growth factor [FGF], EGF, vascular endothelial growth factor [VEGF]). Tube formation was quantified by counting the number of complete tubule branches in four fields of view using ImageJ software (NIH, Bethesda, MD, USA).

qPCR for $\beta$-catenin Real-time quantitative PCR (qPCR) was performed using SYBR Green along with the human WNT Signalling Pathway PCR Array, which contained 84 genes related to Wnt signal transduction (Qiagen, Hilden, NW, USA) using mRNA isolated from $\mathrm{MSC}^{\mathrm{R}}$ vs $\mathrm{MSC}^{\mathrm{NR}}$. See ESM Methods for complete details.
Confocal microscopy and flow cytometry for total $\beta$-catenin MSCs were cultured in six-well plates on glass cover slips and treated with glycogen synthase kinase 3 (GSK3) inhibitor CHIR99021 (EMD Millipore, Billerica, MA, USA) and stained for nuclear $\beta$-catenin. Flow cytometry was used to quantify total cytosolic $\beta$-catenin levels after treatment with CHIR99201. See ESM Methods for complete details.

Human islet culture with MSC CM Human islets from five donors were obtained from the Integrated Islet Distribution Program (IIDP). Upon arrival, 200 islet equivalents were plated in RPMI media without serum (Invitrogen). MSC CM was collected after $24 \mathrm{~h}$ from MSCs treated with DMSO (basal $\mathrm{CM}$ ) or with $10 \mu \mathrm{mol} / 1$ of CHIR99021 (GSK3i CM). CM was concentrated using $3 \mathrm{kDa}$ molecular mass cut-off filters, and $\sim 1.0 \mu \mathrm{g} / \mu \mathrm{l}$ total protein was added to human islet culture for 1, 3 or 7 days (ESM Fig. 1). After islet harvest and dissociation, beta cell content was estimated using FluoZin-3 (Flz3) (ThermoFisher Scientific) and apoptosis was quantified using 7AAD and Annexin-V. To detect islet cell proliferation, $500 \mathrm{nmol} / 1$ of 5 -ethynyl-2'-deoxyuridine (EdU) was added to islet culture $24 \mathrm{~h}$ prior to harvest. Islets were fixed and permeabilised using $10 \%$ formalin and saponin buffer, stained for insulin using a PE-conjugated insulin antibody (R\&D Systems, Minneapolis, MN, USA), and nuclear EdU incorporation was detected using the Click-It flow cytometry assay (Life Technologies). Flow cytometry data were analysed using FloJo software (Treestar, Ashland, OR, USA).

Statistical analysis Statistical analysis was performed using GraphPad Prism version 6.01 (GraphPad, La Jolla, CA, USA) by ANOVA with Tukey's post hoc test or by multiple $t$ tests. Data are expressed as means (SEM).

\section{Results}

Regenerative capacity of human MSCs was donor specific Hyperglycaemic (15-25 mmol/l), STZ-treated ( $35 \mathrm{mg} / \mathrm{kg}$ per day, days 1-5) NOD/SCID mice were i.v. injected on day 10 with MSCs $\left(5 \times 10^{5}\right)[14,18]$, and blood glucose levels were monitored for 42 days. Compared with PBS-injected control mice, MSC samples $(n=3)$ that showed significant reduction in systemic blood glucose from days 17-42 were termed $\mathrm{MSC}^{\mathrm{R}}$ and MSC samples $(n=3)$ that did not show blood glucose reduction ( $>25 \mathrm{mmol} / \mathrm{l}$ ) were termed $\mathrm{MSC}^{\mathrm{NR}}$ (Fig. 1a). There was a significant reduction in blood glucose AUC for $\mathrm{MSC}^{\mathrm{R}}$ vs MSC ${ }^{\mathrm{NR}}$ samples (Fig. 1b). Out of a total of 18 MSC lines tested, six ( $\sim 33 \%)$ were characterised as $\mathrm{MSC}^{\mathrm{R}}$, four $(\sim 22 \%)$ demonstrated intermediate regenerative capacity and eight $(\sim 4 \%)$ were characterised as $\mathrm{MSC}^{\mathrm{NR}}$. MSC lines with intermediate regenerative capacity were not used in this study. All $\mathrm{MSC}^{\mathrm{R}}$ and $\mathrm{MSC}^{\mathrm{NR}}$ lines possessed multipotent 
Fig. 1 Functional characterisation of islet $\mathrm{MSC}^{\mathrm{R}}$ and $\mathrm{MSC}^{\mathrm{NR}}$. (a) MSCs from six donors were injected into STZtreated $(35 \mathrm{mg} / \mathrm{kg}$ per day, days 1-5) NOD/SCID mice on day 10 , and blood glucose was monitored weekly until 42 days to segregate $\mathrm{MSC}^{\mathrm{R}}$ (blue) vs MSC NR (red) samples compared with PBSinjected control mice (black). Blood glucose concentrations were lower after injection of $\mathrm{MSC}^{\mathrm{R}}$ compared with MSC ${ }^{\mathrm{NR}}$ samples. (b) Blood glucose AUC was significantly reduced with $\operatorname{MSC}^{\mathrm{R}}(n=3)$ (blue) vs MSC ${ }^{\mathrm{NR}}$ $(n=3)$ (red) samples. (c) Donor characteristics were similar between $\mathrm{MSC}^{\mathrm{NR}}$ and $\mathrm{MSC}^{\mathrm{R}}$ samples. Data are presented as mean \pm SEM. $* * * p<0.001$ a

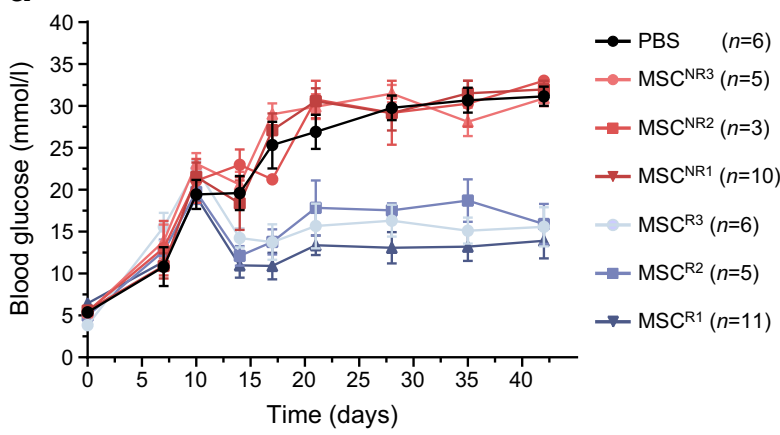

b

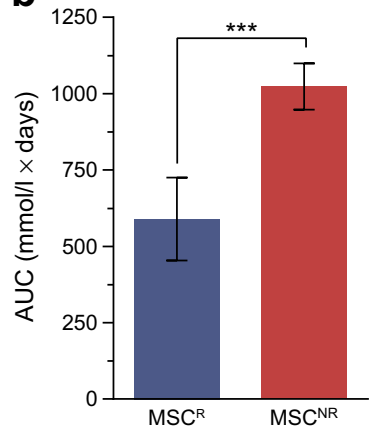

C

\begin{tabular}{lcccc}
\hline Cell line & Age & Sex & Weight $(\mathrm{kg})$ & $\mathrm{BMl}\left(\mathrm{kg} / \mathrm{m}^{2}\right)$ \\
\hline MSC $^{\mathrm{NR} 1}$ & 40 & $\mathrm{M}$ & 103.3 & 31.5 \\
MSC $^{\mathrm{NR2}}$ & 34 & $\mathrm{M}$ & 77.0 & 27.3 \\
MSC $^{\mathrm{NR3}}$ & 29 & $\mathrm{M}$ & 102.0 & 29.5 \\
MSC $^{\mathrm{R} 1}$ & 32 & $\mathrm{M}$ & 71.3 & 24.4 \\
MSC $^{\text {R2 }}$ & 32 & $\mathrm{M}$ & 71.2 & 22.5 \\
MSC $^{\text {R3 }}$ & 54 & $\mathrm{M}$ & 103.0 & 33.6 \\
\hline
\end{tabular}

differentiation potential into adipogenic and osteogenic lineages in vitro [14, 15]. Cell surface phenotype showed that both $\mathrm{MSC}^{\mathrm{R}}$ and $\mathrm{MSC}^{\mathrm{NR}}$ expressed the stromal markers CD90 and CD105 (>95\%) without expression of the pan-leucocyte marker CD45 (data not shown). MSC donor information, including age, sex, weight and BMI, is shown in Fig. 1c.

\section{MSC $^{\mathrm{R}}$ exclusively secreted proteins associated with active} Wnt signalling We have previously shown that $\mathrm{MSC}^{\mathrm{R}}$ demonstrated increased transcription of matrix metalloproteases, EGF-family ligands and downstream products of Wnt signalling [15]. Here, we sought to confirm and identify secreted protein targets that contribute to islet regeneration by comparing the secretome of $\mathrm{MSC}^{\mathrm{R}}$ and $\mathrm{MSC}^{\mathrm{NR}}$ samples using global MS-based proteomics coupled with SILAC. CM was harvested from each MSC line (24 h) and analysed in biological and technical duplicates. The total number of proteins detected for each MSC sample is shown in Fig. 2a. Proteins from each MSC line were combined based on regenerative capacity and complete lists of secreted proteins detected exclusively in $\mathrm{CM}$ from $\mathrm{MSC}^{\mathrm{R}}$ or $\mathrm{MSC}^{\mathrm{NR}}$, or proteins common to both groups, were generated (ESM Tables 2-4). Of 4665 total proteins detected (false discovery rate $1 \%), 3023(\sim 65 \%)$ were produced by both $\mathrm{MSC}^{\mathrm{R}}$ and $\mathrm{MSC}^{\mathrm{NR}}, 850(\sim 18 \%)$ were produced by MSC $^{\mathrm{R}}$ exclusively, while $792(\sim 17 \%)$ were produced by $\mathrm{MSC}^{\mathrm{NR}}$ exclusively (Fig. 2b). Gene ontology using cellular component analysis revealed that MSC ${ }^{\mathrm{R}} \mathrm{CM}$ contained qualitatively more proteins with extracellular localisation $(21 \%$ vs $14 \%$ ), while $\mathrm{MSC}^{\mathrm{NR}} \mathrm{CM}$ contained more membrane-bound proteins (23\% vs 19\%) (Fig. 2c). Moving forward, protein lists were filtered to include only extracellular (secreted) and membrane-bound proteins, and were further analysed for known biological functions and signalling family association.

$\mathrm{MSC}^{\mathrm{R}}$ demonstrated increased representation of secreted proteins associated with angiogenesis and activation of the Wnt-signalling pathway, while MSC $^{\mathrm{NR}}$ showed increased secretion of proteins associated with EGF, FGF and Notch signalling pathways (Fig. 2d). MSC ${ }^{\mathrm{R}}$ exclusively secreted several proteins associated with cell survival and growth, such as FGF7 and bone morphogenetic protein (BMP)4. Proangiogenic factors vascular endothelial growth factor receptor 2 (KDR), vascular endothelial growth factor receptor 4 (FLT4) and regulators of angiogenesis A disintegrin and metalloproteinase with thrombospondin motifs (ADAMTS) 13 and 18 were also unique to the $\mathrm{MSC}^{\mathrm{R}}$ secretome [19]. Notably, $\mathrm{MSC}^{\mathrm{R}}$ secreted potentiators of Wnt signalling (spondins), ligand activators of Wnt signalling (WNT5B) and downstream products of Wnt signalling that modify the extracellular matrix (ECM) (Wntinducible signalling pathway protein 2 [WISP2/CCN5]) (Table 1) [20-22]. In contrast, proteins exclusively detected in $\mathrm{MSC}^{\mathrm{NR}} \mathrm{CM}$ included positive and negative regulators of angiogenesis (FGF receptor (FGFR)4, FLT1) [19, 23], and an abundance of proinflammatory cytokines (IL-6, IL-8) and CX-C motif chemokines (CXCL2, 3, 5) (Table 2) [24]. Collectively, these findings suggested $\mathrm{MSC}^{\mathrm{R}}$ actively secreted cell growth supportive factors with reduced secretion of common proinflammatory signals, and only $\mathrm{MSC}^{\mathrm{R}}$ showed production of multiple effectors associated with active Wnt signalling. 
Fig. 2 Qualitative analyses of proteins exclusively secreted by $\mathrm{MSC}^{\mathrm{R}}$ or $\mathrm{MSC}^{\mathrm{NR}}$. (a) Total proteins identified for $\mathrm{MSC}^{\mathrm{R}}$ (blue) and $\mathrm{MSC}^{\mathrm{NR}}$ (red) lines using SILAC. (b) Venn diagram showing that out of the 4665 total proteins detected, 850 were unique to $\mathrm{MSC}^{\mathrm{R}}$ (blue) and 792 were unique to $\mathrm{MSC}^{\mathrm{NR}}$ (red). (c) $\mathrm{MSC}^{\mathrm{R}}$ demonstrated a higher proportion of proteins with extracellular (secreted) localisation compared with $\mathrm{MSC}^{\mathrm{NR}}$. (d) $\mathrm{MSC}^{\mathrm{R}}$ secreted more proteins associated with the activation of Wnt signalling and angiogenesis. MSC ${ }^{\mathrm{NR}}$ secreted more proteins associated with EGF, FGF and Notch signalling a

\begin{tabular}{lcc}
\hline Cell line & Proteins detected & [Glucose] AUC \\
\hline MSC1 & 2320 & 1022 \\
MSC2 & 2040 & 1014 \\
MSC3 & 1742 & 1032 \\
MSC4 & 1644 & 674 \\
MSC5 & 2154 & 629 \\
MSC6 & 2251 & 529 \\
\hline
\end{tabular}

b

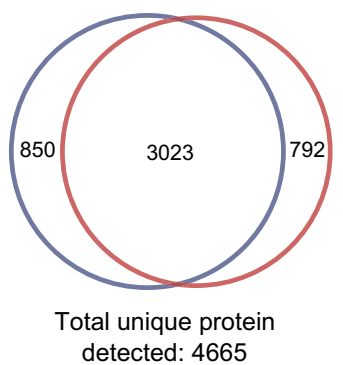

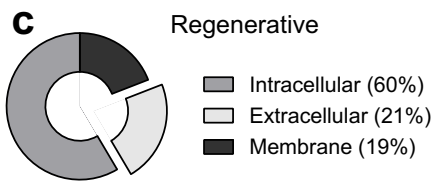

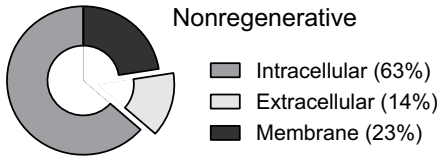

d

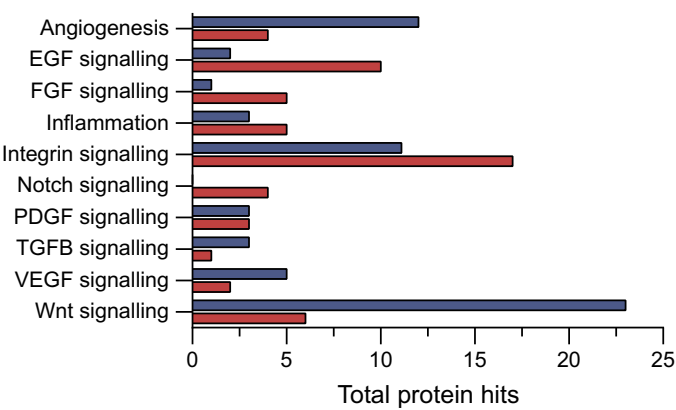

Quantification of Wnt, matrix remodelling and proangiogenic proteins To quantify proteins secreted by both $\mathrm{MSC}^{\mathrm{R}}$ and $\mathrm{MSC}^{\mathrm{NR}}$, quantitative proteomics was performed using label-free quantification $[16,25]$ to identify proteins differentially secreted into serum-containing media. Lists were filtered to include only secreted and membrane-bound proteins, and 1038 common proteins were quantified to generate lists of differentially expressed proteins (ESM Table 5). We did not observe any significant difference in the total number of proteins quantified between samples (Fig. 3a), and 468 proteins were differentially expressed between $\mathrm{MSC}^{\mathrm{R}}$ vs $\mathrm{MSC}^{\mathrm{NR}}$ samples $(p<0.05$, Fig. $3 b)$. Upregulated proteins in $\mathrm{MSC}^{\mathrm{R}}$ $\mathrm{CM}$ included matrix remodelling proteins (matrix metalloproteinase (MMP)1, MMP3, ADAMs and BMPs) [26, 27], effectors of Wnt signalling (WNT5A, secreted frizzled-related protein 1 [SFRP1]) [21], additional targets implicated in angiogenesis (angiogenin [ANG], angiopoietin [ANGPT]1, ANGPTL2, TGFB1, TGFB2, platelet-derived growth factor

Table 1 Exclusively detected proteins in $\mathrm{CM}$ from $\mathrm{MSC}^{\mathrm{R}}$ involved in angiogenesis, inflammation, matrix remodelling and Wnt signalling

\begin{tabular}{lll}
\hline Gene symbol & Protein name & Biological function \\
\hline ADAMTS13 & A disintegrin and metalloproteinase with thrombospondin motifs 13 & Matrix metalloproteinase \\
ADAMTS18 & A disintegrin and metalloproteinase with thrombospondin motifs 18 & Matrix metalloproteinase \\
EGF & Epidermal growth factor & Regulator of cell growth, proliferation and differentiation \\
$F G F 7$ & Fibroblast growth factor 7 & Tissue repair, mitogenic cell survival \\
FLT4 & Vascular endothelial growth factor receptor 3 & Development of vascular networks via secretion of VEFGA/C \\
$I G F 2$ & Insulin-like growth factor 2 & Growth promoting hormone \\
KIT & Mast/stem cell growth factor receptor Kit & Stem cell maintenance, migration, survival and proliferation \\
KDR & Vascular endothelial growth factor receptor 2 & Regulation of angiogenesis and vascular development \\
$M M P 10$ & Stromelysin-2 & Matrix metalloproteinase \\
$M M P 12$ & Macrophage metalloelastase & Matrix metalloproteinase \\
$M M P 3$ & Stromelysin-1 & Matrix metalloproteinase \\
$P D G F D$ & Platelet-derived growth factor D & Regulation of cell proliferation, migration and chemotaxis \\
$S P O N 2$ & Spondin-2 & Activator of Wnt signalling \\
$T I M P 4$ & Metalloproteinase inhibitor 4 & Matrix metalloproteinase inhibitor \\
WISP2 & Wnt1-inducible-signalling pathway protein 2 & CCN5, regulator of cell growth \\
Wht5b & Protein Wnt5b & Activator of Wnt signalling \\
\hline
\end{tabular}


Table 2 Exclusively detected proteins in $\mathrm{CM}$ from $\mathrm{MSC}^{\mathrm{NR}}$ involved in angiogenesis, inflammation, matrix remodelling and Wnt signalling

\begin{tabular}{lll}
\hline $\begin{array}{l}\text { Gene } \\
\text { symbol }\end{array}$ & Protein name & Biological function \\
\hline CXCL2 & C-X-C motif chemokine 2 & \\
CXCL3 & C-X-C motif chemokine 3 & Inflammatory response \\
CXCL5 & C-X-C motif chemokine 5 & Inflammatory response \\
GDF15 & Growth/differentiation factor 15 & Inflammatory response \\
FLT1 & Vascular endothelial growth factor & Regulation of inflammation and apoptosis \\
& receptor 1 & Negative regulator of VEGFA signalling \\
FGFR4 & Fibroblast growth factor receptor 4 & Regulation of angiogenesis and vascular \\
& & development \\
FZD1 & Frizzled-1 & Wnt signalling receptor \\
$F Z D 2$ & Frizzled-2 & Wnt signalling receptor \\
$F Z D 7$ & Frizzled-7 & Wnt signalling receptor \\
$I L-1 B$ & Interleukin-1 beta & Inflammatory response \\
$I L-6$ & Interleukin-6 & Inflammatory response \\
$I L-8$ & Interleukin-8 & Inflammatory response \\
$M M P 11$ & Stromelysin-3 & Matrix metalloproteinase \\
$N O V$ & Protein NOV homologue & CCN3, promoter of cell differentiation \\
$T G F B R 2$ & TGF-beta receptor type-2 & Regulator of cell growth, proliferation \\
& & and differentiation \\
\hline & &
\end{tabular}

receptor [PDGFR]A, B) [23, 28] and chemokines involved in cell recruitment (CXCL12) (Fig. 3c) [28, 29]. Thus, the activation of Wnt and proangiogenic signalling again correlated with islet regenerative potency. Conversely, the negative regulator of Wnt signalling Dickkopf-related protein (DKK)1 was increased 2 .5-fold in $\mathrm{MSC}^{\mathrm{NR}} \mathrm{CM}$, and negative regulators of angiogenesis (ADAMTSs, thrombospondin [THBS]1 and THBS2) and inhibitors of matrix remodelling proteins (metalloproteinase inhibitor [TIMP]3) were increased in $\mathrm{MSC}^{\mathrm{NR}} \mathrm{CM}$ [30]. HMVECs were cultured on growth factor-reduced Geltrex to assess the angiogenic potential of MSC CM in vitro (Fig. 3e). A significant increase in the tubule formation was observed when HMVECs were cultured using CM from MSC $^{\mathrm{R}}$ compared with MSC ${ }^{\mathrm{NR}}$. Representative photomicrographs of tube formation after $24 \mathrm{~h}$ are shown in ESM Fig. 2. Finally, we identified that pro-differentiation proteins associated with Notch signalling were upregulated in $\mathrm{MSC}^{\mathrm{NR}}$ (NOTCH2, JAG-1) [31], suggesting that inhibited Wnt and angiogenic signalling and activated Notch signalling was associated with diminished islet regenerative capacity.

\section{MSC $^{\mathrm{R}}$ increased expression of Wnt pathway mRNA To} confirm the activation of Wnt signalling in $\mathrm{MSC}^{\mathrm{R}}$, we assessed mRNA expression of 84 genes related to Wnt pathway signal transduction, using three $\mathrm{MSC}^{\mathrm{R}}$ and three $\mathrm{MSC}^{\mathrm{NR}}$ lines performed in duplicate. Transcripts with differential expression greater than twofold between $\mathrm{MSC}^{\mathrm{R}}$ vs $\mathrm{MSC}^{\mathrm{NR}}$ revealed 18 significantly changing genes (ESM Fig. 3a,b), normalised to the geometric mean of three housekeeping genes: $A C T B, B 2 M$ and HPRT1, that did not show significant variation across samples. Notably, all differentially expressed mRNAs (18 genes) were upregulated in $\mathrm{MSC}^{\mathrm{R}}$, and no significantly changing genes were downregulated. Upregulation of $W N T 5 A / B$ in $\mathrm{MSC}^{\mathrm{R}}$ were confirmed at the mRNA level. Other upregulated mRNA in $\mathrm{MSC}^{\mathrm{R}}$ included transcription factors (FOSL1, JUN) and receptors associated with Wnt signalling (FZD5) [20, 32]. Taken together, upregulation of Wnt signalling at the mRNA and protein level strongly suggests active Wnt signalling during expansion was a unique and conserved characteristic that correlated with the reduction of hyperglycaemia after transplantation of $\mathrm{MSC}^{\mathrm{R}}$ in vivo.

MSCs show activation of Wnt signalling via accumulation of nuclear $\beta$-catenin Canonical Wnt signalling converges on the actions of $\beta$-catenin, a transcription factor that increases the expression of downstream effectors of Wnt signals [33]. We mimicked Wnt signalling in $\mathrm{MSC}^{\mathrm{NR}}$ and $\mathrm{MSC}^{\mathrm{R}}$ using a small molecule (CHIR99201) inhibitor of GSK3. GSK3 actively phosphorylates $\beta$-catenin, which marks it for ubiquitination and degradation. Therefore, inhibition of GSK3 leads to stabilisation and accumulation of free $\beta$ catenin [20]. MSC samples showed maximal increases in total $\beta$-catenin using 5-10 $\mu \mathrm{mol} / \mathrm{l}$ CHIR99201 for 24-48 h. We then used confocal microscopy to visualise the accumulation of nuclear $\beta$-catenin (Fig. 4). Compared with DMSO control cells (Fig. 4a), MSC ${ }^{\mathrm{NR}}$ and $\mathrm{MSC}^{\mathrm{R}}$ stimulated with $10 \mu \mathrm{mol} / \mathrm{l}$ CHIR99201 showed increased nuclear $\beta$-catenin localisation (Fig. 4b, c). Next, MSC ${ }^{\mathrm{NR}}$ and $\mathrm{MSC}^{\mathrm{R}}$ samples were analysed in quadruplicate to quantify total $\beta$-catenin levels by flow cytometry (Fig. 4d). Representative dot plots are shown in 


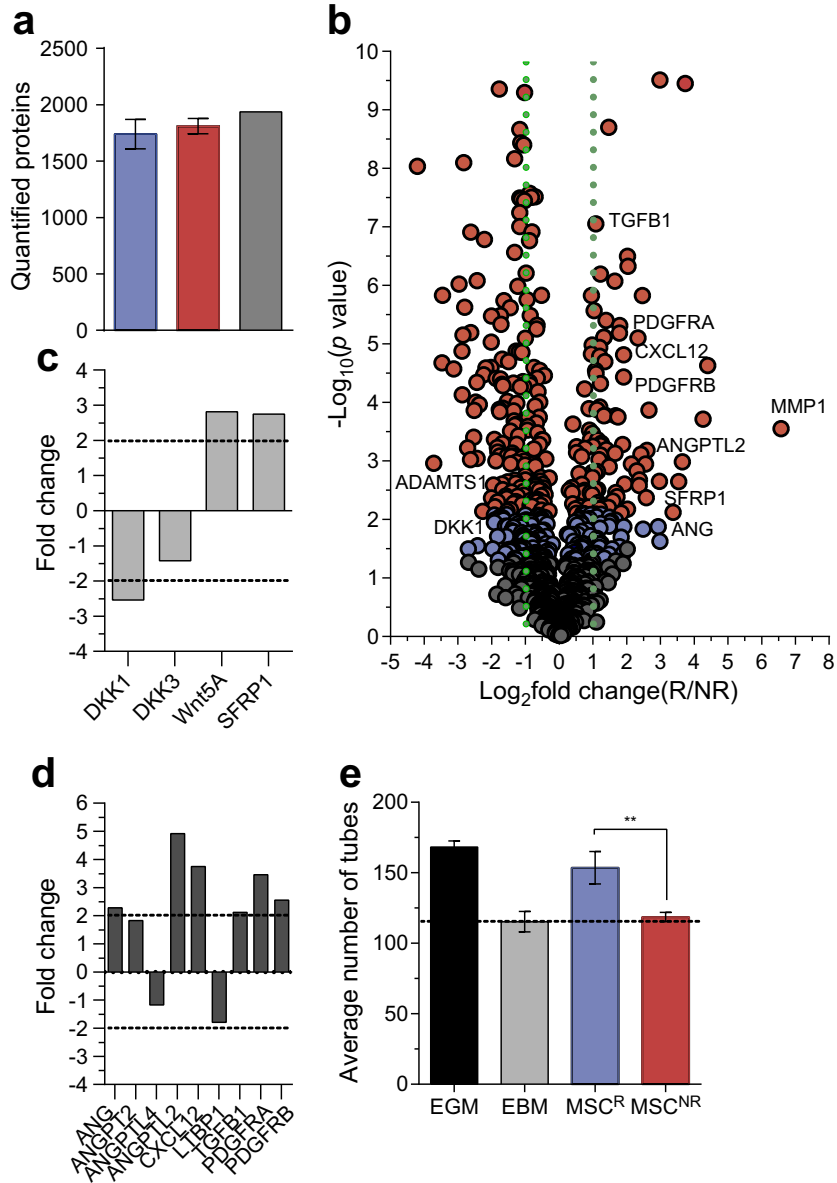

Fig. 3 Quantitative analyses of proteins secreted by both $\mathrm{MSC}^{\mathrm{R}}$ and $\mathrm{MSC}^{\mathrm{NR}}$. (a) Total number of proteins quantified within all three $\mathrm{MSC}^{\mathrm{R}}$ (blue), $\mathrm{MSC}^{\mathrm{NR}}$ (red) samples and total number of quantified proteins (grey). (b) Representative volcano plot of differentially expressed secreted proteins. A change greater than twofold is represented outside the green boundaries. Red, $p<0.01$; blue, $p<0.05$; grey, $p>0.05$. (c) $\mathrm{MSC}^{\mathrm{R}}$ showed increased secretion of Wnt activators (Wnt5A), while $\mathrm{MSC}^{\mathrm{NR}}$ showed increased secretion of Wnt inhibitors (DKK1). (d) $\mathrm{MSC}^{\mathrm{R}}$ demonstrated increased secretion of proangiogenic proteins, TGFB1 and SDF-1 (CXCL12). (e) Spontaneous tubule formation of HMVECs on growth factor-reduced Geltrex was increased when cultured using $\mathrm{CM}$ generated from $\mathrm{MSC}^{\mathrm{R}}$ compared with $\mathrm{MSC}^{\mathrm{NR}}$. Data are presented as mean $\pm \mathrm{SD} . * * p<0.01$

ESM Fig. 4. Compared with DMSO control cells, both MSC subtypes treated with CHIR99201 showed significantly increased total $\beta$-catenin. Thus, inhibition of GSK3 with CHIR99201 mimicked activated Wnt signalling in $\mathrm{MSC}^{\mathrm{R}}$ and $\mathrm{MSC}^{\mathrm{NR}}$.

\section{GSK3 inhibition in MSC $^{\mathrm{NR}}$ generates $\mathrm{CM}$ that improves} human beta cell survival in vitro To assess whether $\mathrm{MSC}^{\mathrm{R}}$ $\mathrm{CM}$ could improve beta cell survival in vitro, we cultured primary human islet preparations for up to 7 days in $\mathrm{CM}$ from $\mathrm{MSC}^{\mathrm{R}}$ and $\mathrm{MSC}^{\mathrm{NR}}$, and performed multiparametric flow cytometry to analyse beta cell survival and proliferation. Human islets were obtained through the IIDP. Compared with $\mathrm{MSC}^{\mathrm{NR}}$
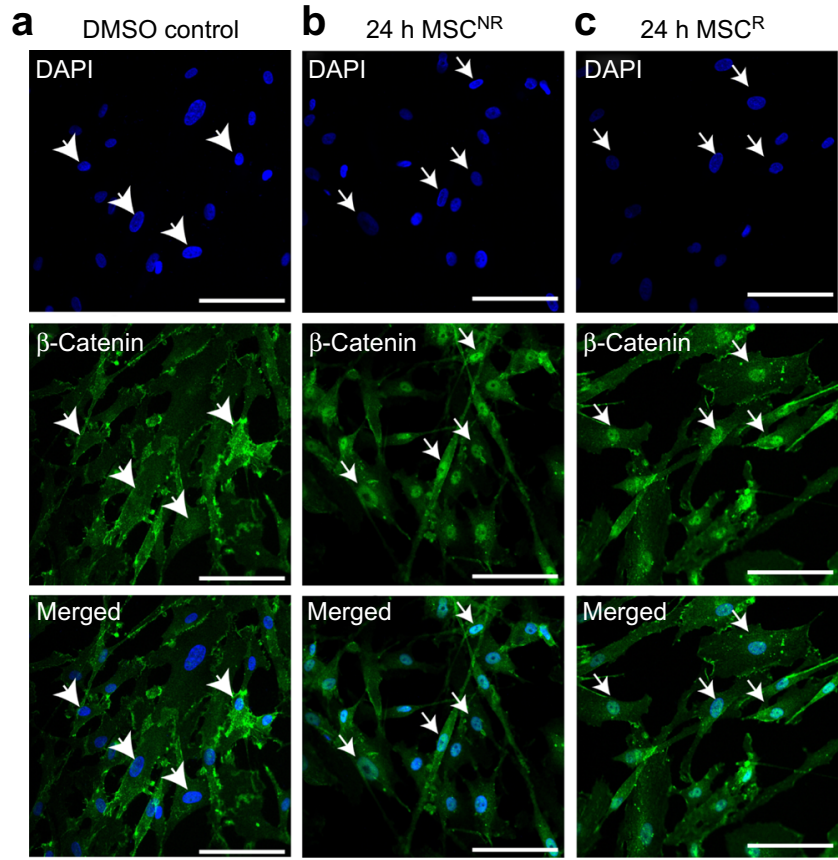

d

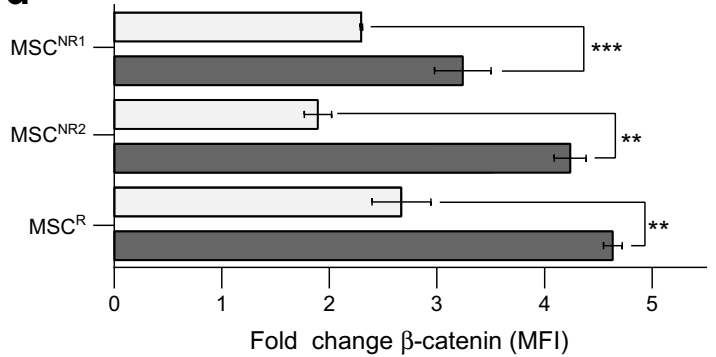

Fig. 4 MSC treatment with GSK3 inhibitor induced nuclear $\beta$-catenin localisation. Representative photomicrographs showing $\mathrm{MSC}^{\mathrm{R}}$ and $\mathrm{MSC}^{\mathrm{NR}}$ stained for $\beta$-catenin (green) and DAPI (blue) after treatment with (a) DMSO vehicle, (b) CHIR99201 (24 h) MSC $^{\mathrm{NR}}$, (c) CHIR99201 (24 h) MSC ${ }^{\mathrm{R}}$. White arrows indicate examined nuclei with nuclear staining and arrowheads indicate examined nuclei without nuclear staining. Scale bars, $50 \mu \mathrm{m}$. (d) Intracellular $\beta$-catenin was increased in both $\mathrm{MSC}^{\mathrm{R}}$ and $\mathrm{MSC}^{\mathrm{NR}}$ treated with CHIR99201 (grey bars) or DMSO control (white bars). Data are presented as mean \pm SEM. $* * p<0.01$, $* * * p<0.001$

$\mathrm{CM}$, human islets grown in $\mathrm{MSC}^{\mathrm{R}} \mathrm{CM}$ showed increased total number of beta cells (Fig. 5a) and an increased frequency of live beta cells (Fig. 5b) after 7 days of culture. To further assess the influence of active Wnt signalling on islet regenerative paracrine function, we also assessed whether CM generated by MSC $^{\mathrm{NR}}$ treated with a GSK3 inhibitor (CHIR99201) during expansion could promote human beta cell survival or proliferation in cultured human islets. CM recovered from $\mathrm{MSC}^{\mathrm{NR}}$ cultured with vehicle control (DMSO) only (basal $\mathrm{CM}$ ) was compared with $\mathrm{CM}$ recovered from $\mathrm{MSC}^{\mathrm{NR}}$ treated with $10 \mu \mathrm{mol} / 1$ CHIR99201 (GSK3i CM) (Fig. 5c). Five independent human islet samples were cultured as indicated in ESM Fig. 1. At each time point, human islets were harvested, dissociated with trypsin, and stained using Flz3 to estimate 
beta cell frequency, 7AAD to determine cell viability and Annexin- $\mathrm{V}$ to determine apoptosis rates by multiparametric flow cytometry. Representative dot plots are shown in ESM Fig. 5. Compared with basal CM, human islets grown in CM generated by GSK3-inhibited MSC ${ }^{\mathrm{NR}}$ showed increased total cell numbers (Fig. 5d), beta cell numbers (Fig. 5e) and the proportion of live (7AAD-) beta cells at 7 days culture (Fig. 5f). However, no significant changes in the frequency of apoptotic beta cells were observed (Fig. $5 \mathrm{~g}$ ).

To determine whether increased beta cell number was augmented by increased beta cell proliferation in vitro, we measured EdU incorporation in insulin + beta cells. At each time point, human islets were harvested, permeabilised, stained for intracellular insulin and proliferation was detected by EdU incorporation using the Click-It system. Representative dot plots showing EdU+ cell detection for each condition are shown in ESM Fig. 6. Compared with basal CM, islet exposure to CM generated by GSK3-inhibited MSC ${ }^{\mathrm{NR}}$ increased the overall frequency of proliferating cells (Fig. 6a) and proportion of total proliferating beta cells (Fig. 6b). Finally, islet donor variability was documented by donor information including age, sex, BMI and average blood glucose levels (Fig. 6c). Collectively, these data suggest that $\mathrm{CM}$ generated from MSC $^{\mathrm{NR}}$ treated with CHIR99201 promoted beta cell survival and induced beta cell proliferation in vitro.

\section{Discussion}

We used comprehensive SILAC-based proteomic analyses to identify MSC-secreted factors that correlated with the capacity to lower circulating blood glucose after transplantation into STZ-treated NOD/SCID mice. $\mathrm{MSC}^{\mathrm{R}}$, demonstrated glucose-lowering capacity after transplantation, secreted proteins associated with cell growth, matrix remodelling, immunosuppressive and proangiogenic properties. In contrast, $\mathrm{MSC}^{\mathrm{NR}}$, which lacked islet regenerative functions, secreted proteins involved in the initiation of inflammation and the negative regulation of angiogenesis. Notably, $\mathrm{MSC}^{\mathrm{R}}$ consistently demonstrated mRNA and protein expression associated with active Wnt signalling, a novel signature that correlated with islet regenerative capacity. Inhibition of GSK3 activity with CHIR99201 mimicked Wnt signalling in $\mathrm{MSC}^{\mathrm{NR}}$ and resulted in the generation of $\mathrm{CM}$ that supported beta cell
Fig. 5 Culture of human islets in GSK-inhibited CM increased beta cell number. Human islets cultured in $\mathrm{CM}$ generated from $\mathrm{MSC}^{\mathrm{R}}$ showed (a) increased beta cell number and (b) increased proportion of live beta cells after 7 days of culture. (c) Human islets were cultured in $\mathrm{CM}$ generated by $\mathrm{MSC}^{\mathrm{NR}}$ treated with DMSO (basal CM) or CHIR99201 (GSK3i CM). Compared with human islets cultured in basal CM (red), islets cultured in GSK3inhibited CM (blue) showed increased (d) cell number, (e) beta cell number and (f) proportion of live beta cells at day 7. (g) Human islets cultured in $\mathrm{MSC}^{\mathrm{NR}} \mathrm{CM}$ did not alter apoptotic beta cell frequency. Data are presented as mean \pm SEM. $* p<0.05$, $* * p<0.01, * * * p<0.001$ a

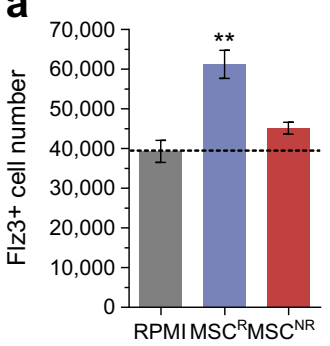

d

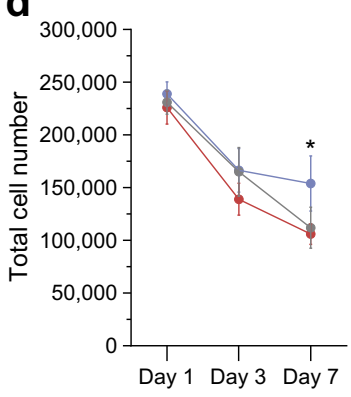

f

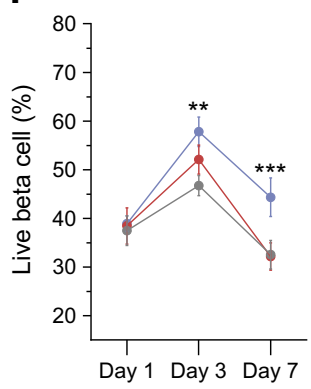

b

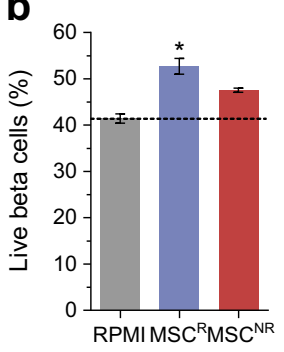

e

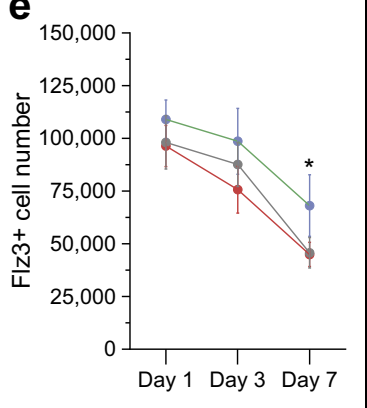

g

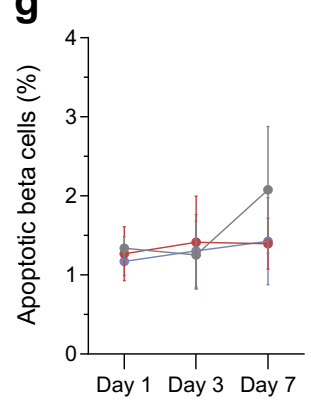

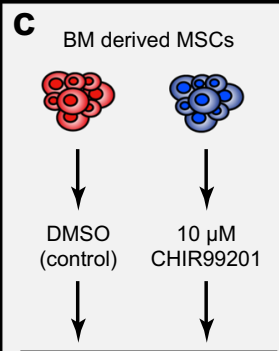

Collect and concentrate conditioned media $10 X$ Quantify and equalise protein amount

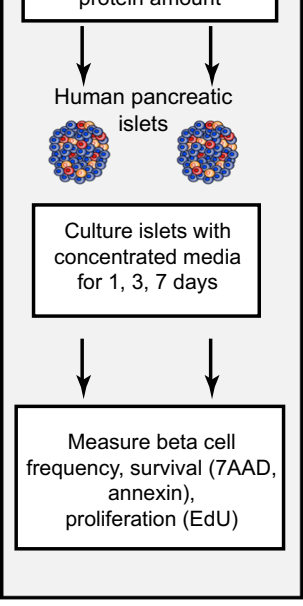
Measure beta cell
frequency, survival (7AAD annexin), 
Fig. 6 Culture of human islets in GSK-inhibited CM increased beta cell proliferation. (a, b)

Compared with human islets cultured in basal CM (red), islets cultured in GSK3i CM (blue) showed increased proportion of insulin+/EdU+ beta cells at day 3 . (c) Islets were obtained through the IIDP from five independent donors with variable weight, BMI and average blood glucose (Avg.BG) values. Data are presented as mean \pm SEM. $* p<0.05$ a

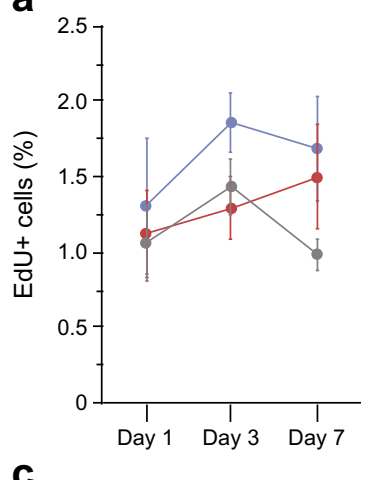

b

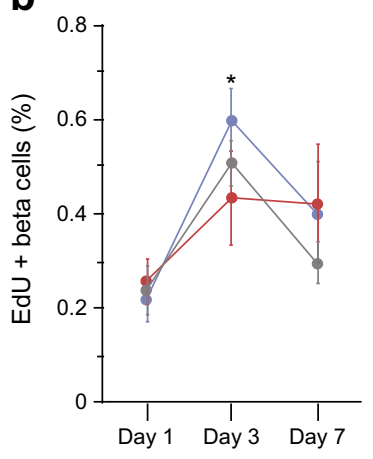

\begin{tabular}{lccccc}
\hline Isolation ID & Age & Sex & Weight $(\mathrm{kg})$ & BMI $\left(\mathrm{kg} / \mathrm{m}^{2}\right)$ & Avg.BG (mmol/l) \\
\hline UWHI263R & 53 & M & 107.0 & 31.10 & 10.5 \\
H585A & 29 & F & 101.0 & 35.80 & 10.8 \\
HP-16206-01 & 30 & M & 133.8 & 35.90 & 9.8 \\
HP-16250-01 & 32 & F & 61.7 & 24.90 & 6.4 \\
UWHI267R & 37 & M & 95.0 & 27.60 & 9.8 \\
\hline
\end{tabular}

survival and proliferation within cultured human islets in vitro. Thus, we report a central role for Wnt signalling in the establishment of an islet regenerative secretory profile in $\mathrm{MSC}^{\mathrm{R}}$, and improve our understanding of MSC-secreted signals governing islet regeneration. We predict this dataset will aid in the development of future therapies to augment islet regeneration during diabetes.

Functional beta cells secrete proangiogenic proteins responsible for recruiting circulating or tissue resident progenitor cells to islets after damage [34]. However, during autoimmunity or after islet isolation and transplantation these critical functions are likely to be dysregulated, resulting in transient ischaemia that significantly impairs islet function [35]. Increased production of potent proangiogenic factors, such as FGF7, PDGF and VEGFA, by MSC ${ }^{\mathrm{R}}$ underscore the functional capacity to generate a proangiogenic microenvironment. Collectively these proteins potently stimulate human islet vascularisation in vitro [36]. To validate the functional relevance of proangiogenic secretory patterns, $\mathrm{MSC}^{\mathrm{R}} \mathrm{CM}$ showed increased capacity to induce spontaneous tubule formation by HMVEC compared with $\mathrm{MSC}^{\mathrm{NR}} \mathrm{CM}$. Interestingly, $\mathrm{MSC}^{\mathrm{NR}}$ primarily secreted negative regulators of angiogenesis such as FLT1 and FGFR4, which have both been shown to inhibit the signal cascade mediated by VEGFA [19]. We have previously shown that intrapancreatic transplantation of proangiogenic haematopoietic progenitor cells induced islet revascularisation and beta cell proliferation and augmented systemic insulin release in STZ-treated NOD/ SCID mice [15]. Therefore, our data suggest $\mathrm{MSC}^{\mathrm{R}}$ may also formulate a proangiogenic microenvironment to support beta cell survival and function within endogenous or transplanted islets.

The prevention of proinflammatory states within damaged islets may also be relevant in the context of MSC therapy for type 1 diabetes. The presence of central mediators of inflammation in CM from MSC ${ }^{\mathrm{NR}}$, such as IL- $1 \beta$, IL-6 and IL-8, suggests that $\mathrm{MSC}^{\mathrm{NR}}$ may contribute to a proinflammatory cascades. In people with type 1 diabetes, beta cell destruction is initiated by a combination of proinflammatory cytokines, including IL-1 $\beta$ and IL-6 [37], and CXCL family chemokines, leading to recruitment of immune effectors that mediate beta cell destruction [38]. Furthermore, high levels of IL-8 have been linked to elevated NEFA, which can signal inflammatory cascades in the pancreas [39]. In contrast, these cytokines and chemokines were not secreted by $\mathrm{MSC}^{\mathrm{R}}$. Rather, $\mathrm{MSC}^{\mathrm{R}}$ secreted cytokines that could potentially reduce inflammation. For example, TGF- $\beta$ suppresses the secretion of various inflammatory cytokines/chemokines, and induces cytokine secretion patterns that balance local immunity [40]. Stromal cell-derived factor 1 (CXCL12), also upregulated by $\mathrm{MSC}^{\mathrm{R}}$, has been shown to directly promote beta cell survival through the activation of AKT [41]. Collectively, we propose $\mathrm{MSC}^{\mathrm{R}}$ generate a niche with reduced inflammation, improving beta cell survival, while $\mathrm{MSC}^{\mathrm{NR}}$ contribute to a more deleterious proinflammatory microenvironment.

Inadequate beta cell mass leads to hyperglycaemia in both type 1 and type 2 diabetes. Currently, there is significant interest in restoration of beta cell mass through induction of endogenous regenerative mechanisms in situ 
using cellular or protein therapies. Our previous transplantation studies suggested that human BM-derived MSCs stimulate an islet regeneration programme with neogenic characteristics. Mice transplanted with $\mathrm{MSC}^{\mathrm{R}}$, demonstrated improved glycaemic control via the emergence of small beta cell clusters associated with the ductal epithelium [14]. Several identified effectors may act in synergy to mediate islet regenerative processes. First, $\mathrm{MSC}^{\mathrm{R}}$ showed increased secretion of EGF previously shown to increase beta cell mass in rodents by stimulating beta cell replication [42]. However, EGF receptor signalling was required for expansion of murine beta cell mass in response to a high-fat diet, but was not crucial for neoislet formation after pancreatic ductal ligation [43]. Second, the CCN family of extracellular matrix-associated, heparin-binding proteins has been shown to modulate cell growth and repair in many tissues by increasing the bioavailability of BMPs, VEGF, Wnt and TGF ligands [44]. More specifically, connective tissue growth factor (CTGF) or CCN2 has been widely studied during beta cell development [45]. Within our dataset, $\mathrm{MSC}^{\mathrm{R}}$ exclusively secreted CCN5). Previously, we have identified WISP1 (encoding CCN4) and WISP2 (encoding CCN5) mRNA as being upregulated in $\mathrm{MSC}^{\mathrm{R}}$ [15]. Importantly, CCN4 and CCN5 secretion is directly linked to active Wnt signalling. CCN5 also represents a link between insulin and IGF-I regulation in islet function, and CCN5 overexpression leads to increased islet cell growth in vitro [46]. Interestingly, $\mathrm{MSC}^{\mathrm{NR}}$ exclusively secreted nephroblastoma overexpressed protein or NOV isoform (CCN3), which has been shown to impair beta cell proliferation and inhibit glucose-stimulated insulin secretion in vitro [45]. Thus, we predict modification of the ECM via Wnt-inducible CCN protein regulation is a critical step in islet regenerative processes.

One of the key differences between $\mathrm{MSC}^{\mathrm{R}}$ and $\mathrm{MSC}^{\mathrm{NR}}$ was the activation of Wnt signalling. Wnt signalling is highly conserved in primitive MSCs and is involved in multiple developmental processes, including cell proliferation, growth and fate determination. Aly and colleagues have shown that delivery of WNT3A and R-spondin to cultured islets increased the proliferation of adult human beta cells [47]. Our data suggests that $\mathrm{MSC}^{\mathrm{R}}$ propagate Wnt signalling primarily by autocrine secretion of WNT5A/B ligands with potential regulation by SFRP1. In contrast, MSC ${ }^{\mathrm{NR}}$ expressed proteins that inhibit Wnt signalling, namely DKK1 and DKK3. Although more research is required to determine potential effects of Wnt5 signalling on human beta cells, it is clear that $\mathrm{MSC}^{\mathrm{R}}$ maintain active canonical Wnt signalling and increase secretion of downstream effectors that promote beta cell regeneration. By inhibiting GSK3 activity using CHIR99201, we have shown that the activation of canonical Wnt pathway signals in $\mathrm{MSC}^{\mathrm{NR}}$ can mimic Wnt signalling allowing accumulation of nuclear $\beta$ - catenin. Human pancreatic islets grown in media conditioned by MSC $^{\mathrm{NR}}$ treated with CHIR99201 showed increased beta cell survival and proliferation during 7 days of culture. Manipulation of Wnt signalling through the inhibition of GSK3 has been previously suggested to directly increase beta cell proliferation. One study treated diabetic neonates with $\mathrm{LiCl}$, a known inhibitor of GSK3, and doubled beta cell mass in rat models of diabetes [48]. Others have inhibited GSK3 using small molecules to regulate islet cell survival and proliferation in vitro [49]. Although, the mechanisms by which inhibition of GSK3 affects beta cell survival and proliferation in vivo is still unclear, our results suggest that activation of Wnt signalling in pancreasresident stromal cells may have practical application in beta cell regenerative therapies. Nonetheless, activation of Wnt signalling during MSC culture increased the regenerative capacity of $\mathrm{MSC}^{\mathrm{NR}}$ by altering downstream secretory patterns.

In summary, our data outlines several dynamic and complementary pathways that formulate a regenerative microenvironment applicable to the development of islet expansion therapies for diabetes using MSCs or their secretory products. The proteomic data reported in this study will be used in future studies to characterise functional mechanisms relevant to islet regeneration, or as screening technology to select MSC subpopulations that possess augmented capacity to regenerate islets in situ.

Acknowledgements We wish to acknowledge P. Pittock (Don Rix Protein Identification Facility, Department of Biochemistry, Western University) for troubleshooting, maintenance and care of the MS instrumentation and for insightful input on method optimisation.

Data availability Proteomic raw data files that support the findings of this study are available from the corresponding author upon reasonable request.

Funding This study was supported in part by a Natural Sciences and Engineering Research Council of Canada (NSERC, R-3095A03) grant to GAL, and a JDRF USA Strategic Research Agreement on Optimizing Beta Cell Regeneration (JDRF, 2-SRA-2015-60-Q-R) grant to DAH.

Duality of interest The authors declare that there is no duality of interest associated with this manuscript.

Contribution statement MK performed experiments, presented and analysed the data, and wrote and critically revised the manuscript and approved the final version. GIB performed in vivo characterisation experiments, collected and assembled data, and provided critical revision of the manuscript and final approval. SES performed confocal imaging, collected and assembled data, and provided critical revision and final approval of the manuscript. GAL and DAH designed the study, provided financial support and data interpretation, wrote and reviewed the manuscript and provided final approval. DAH is responsible for the integrity of the work as a whole. 


\section{References}

1. Olokoba AB, Obateru OA, Olokoba LB (2012) Type 2 diabetes mellitus: a review of current trends. Oman Med J 27:269-273

2. Ryan EA, Paty BW, Senior PA et al (2005) Five-year follow-up after clinical islet transplantation. Diabetes 54:2060-2069

3. Shapiro AMJ, Ryan EA, Lakey JRT (2001) Islet cell transplantation. Lancet 358:S214

4. Qi C, Yan X, Huang C et al (2015) Biomaterials as carrier, barrier and reactor for cell-based regenerative medicine. Protein Cell 6: 638-653

5. Pomeransky AA, Khriplovich IB (1999) Equations of motion of spinning relativistic particle in external fields. Surv High Energy Phys 14:145-173

6. Lee RH, Seo MJ, Reger RL et al (2006) Multipotent stromal cells from human marrow home to and promote repair of pancreatic islets and renal glomeruli in diabetic NOD/scid mice. Proc Natl Acad Sci 103:17438-17443

7. Lai RC, Arslan F, Lee MM et al (2010) Exosome secreted by MSC reduces myocardial ischemia/reperfusion injury. Stem Cell Res 4: 214-222

8. Anderson JD, Johansson HJ, Graham CS et al (2016) Comprehensive proteomic analysis of mesenchymal stem cell exosomes reveals modulation of angiogenesis via nuclear factorKappab signaling. Stem Cells 34:601-613

9. Meyerrose T, Olson S, Pontow S et al (2010) Mesenchymal stem cells for the sustained in vivo delivery of bioactive factors. Adv Drug Deliv Rev 62:1167-1174

10. Madec AM, Mallone R, Afonso G et al (2009) Mesenchymal stem cells protect NOD mice from diabetes by inducing regulatory $\mathrm{T}$ cells. Diabetologia 52:1391-1399

11. Berman DM, Willman MA, Han D et al (2010) Mesenchymal stem cells enhance allogeneic islet engraftment in nonhuman primates. Diabetes 59:2558-2568

12. Karnieli O, Izhar-Prato Y, Bulvik S, Efrat S (2007) Generation of insulin-producing cells from human bone marrow mesenchymal stem cells by genetic manipulation. Stem Cells 25:2837-2844

13. Urbán VS, Kiss J, Kovács J et al (2008) Mesenchymal stem cells cooperate with bone marrow cells in therapy of diabetes. Stem Cells 26:244-253

14. Bell GI, Broughton HC, Levac KD et al (2012a) Transplanted human bone marrow progenitor subtypes stimulate endogenous islet regeneration and revascularization. Stem Cells Dev 21:97-109

15. Bell GI, Meschino MT, Hughes-Large JM et al (2012b) Combinatorial human progenitor cell transplantation optimizes islet regeneration through secretion of paracrine factors. Stem Cells Dev 21:1863-1876

16. Ong S-E (2002) Stable isotope labeling by amino acids in cell culture, SILAC, as a simple and accurate approach to expression proteomics. Mol Cell Proteomics 1:376-386

17. Bendall SC, Hughes C, Stewart MH et al (2008) Prevention of amino acid conversion in SILAC experiments with embryonic stem cells. Mol Cell Proteomics 7:1587-1597

18. Hess D, Li L, Martin M et al (2003) Bone marrow-derived stem cells initiate pancreatic regeneration. Nat Biotechnol 21:763-770

19. Shibuya M (2011) Vascular endothelial growth factor (VEGF) and its receptor (VEGFR) signaling in angiogenesis: a crucial target for anti- and pro-angiogenic therapies. Genes Cancer 2:1097-1105

20. Gordon MD, Nusse R (2006) Wnt signaling: multiple pathways, multiple receptors, and multiple transcription factors. J Biol Chem 281:22429-22433

21. Yang Y (2003) Wnt5a and Wnt5b exhibit distinct activities in coordinating chondrocyte proliferation and differentiation. Development 130:1003-1015
22. Bakondi B, Shimada IS, Peterson BM, Spees JL (2011) SDF-1 $\alpha$ secreted by human CD133-derived multipotent stromal cells promotes neural progenitor cell survival through CXCR7. Stem Cells Dev 20:1021-1029

23. Huang Z, Bao SD (2004) Roles of main pro- and anti-angiogenic factors in tumor angiogenesis. World J Gastroenterol 10:463-470

24. Aziz M, Jacob A, Yang W-L et al (2013) Current trends in inflammatory and immunomodulatory mediators in sepsis. J Leukoc Biol 93:329-342

25. Cox J, Hein MY, Luber CA, Paron I (2014) Accurate proteomewide label-free quantification by delayed normalization and maximal peptide ratio extraction, termed MaxLFQ. Mol Cell 13:25132526

26. Rohani MG, Parks WC (2015) Matrix remodeling by MMPs during wound repair. Matrix Biol 44-46:113-121

27. Vadon-Le Goff S, Hulmes DJ, Moali C (2015) BMP-1/tolloid-like proteinases synchronize matrix assembly with growth factor activation to promote morphogenesis and tissue remodeling. Matrix Biol 44-46:14-23

28. Boomsma RA, Geenen DL (2012) Mesenchymal stem cells secrete multiple cytokines that promote angiogenesis and have contrasting effects on chemotaxis and apoptosis. PLoS One 7:e35685

29. Ho TK, Tsui J, Xu S et al (2010) Angiogenic effects of stromal cellderived factor-1 (SDF-1/CXCL12) variants in vitro and the in vivo expressions of CXCL12 variants and CXCR4 in human critical leg ischemia. J Vasc Surg 51:689-699

30. Pepper MS (1996) Positive and negative regulation of angiogenesis: from cell biology to the clinic. Vasc Med 1:259-266

31. Blanpain C, Lowry WE, Pasolli HA, Fuchs E (2006) Canonical notch signaling functions as a commitment switch in the epidermal lineage. Genes Dev 20:3022-3035

32. Yokoyama N, Yin D, Malbon CC (2007) Abundance, complexation, and trafficking of $\mathrm{Wnt} / \beta$-catenin signaling elements in response to Wnt3a. J Mol Signal 2:11

33. Meijer L, Flajolet M, Greengard P (2004) Pharmacological inhibitors of glycogen synthase kinase 3. Trends Pharmacol Sci 25:471480

34. Jin DK, Shido K, Kopp H et al (2006) Cytokine-mediated deployment of SDF-1 induces revascularization through recruitment of CXCR4+ hemangiocytes. Nat Med 12:557-567

35. Brissova M, Shostak A, Shiota M et al (2006) Pancreatic islet production of vascular endothelial growth factor-A is essential for islet vascularization, revascularization, and function. Diabetes 55:2974 2985

36. Luo JZQ, Xiong F, Al-Homsi AS et al (2011) Human BM stem cells initiate angiogenesis in human islets in vitro. Bone Marrow Transplant 46:1128-1137

37. Blandino-Rosano M, Perez-Arana G, Mellado-Gil JM et al (2008) Anti-proliferative effect of pro-inflammatory cytokines in cultured beta cells is associated with extracellular signal-regulated kinase $1 / 2$ pathway inhibition: protective role of glucagon-like peptide-1. J Mol Endocrinol 41:35-44

38. Eizirik DL, Colli ML, Ortis F (2009) The role of inflammation in insulitis and beta-cell loss in type 1 diabetes. Nat Rev Endocrinol 5: 219-226

39. Shi H, Kokoeva MV, Inouye K et al (2006) TLR4 links innate immunity and fatty acid - induced insulin resistance. J Clin Invest 116:3015-3025

40. Sanjabi S, Zenewicz LA, Kamanaka M, Flavell RA (2009) Antiinflammatory and pro-inflammatory roles of TGF- $\beta$, IL-10, and IL22 in immunity and autoimmunity. Curr Opin Pharmacol 9:447453

41. Yano T, Liu Z, Donovan J et al (2007) Stromal cell-derived factor-1 (SDF-1)/CXCL12 attenuates diabetes in mice and promotes pancreatic beta-cell survival by activation of the prosurvival kinase Akt. Diabetes 56:2946-2957 
42. Rooman I, Bouwens L (2004) Combined gastrin and epidermal growth factor treatment induces islet regeneration and restores normoglycaemia in $\mathrm{C} 57 \mathrm{BI} / \mathrm{J}$ mice treated with alloxan. Diabetologia 47:259-265

43. Hakonen E, Ustinov J, Mathijs I et al (2011) Epidermal growth factor (EGF)-receptor signalling is needed for murine beta cell mass expansion in response to high-fat diet and pregnancy but not after pancreatic duct ligation. Diabetologia 54:1735-1743

44. Zuo GW, Kohls CD, He BC et al (2010) The CCN proteins: important signaling mediators in stem cell differentiation and tumorigenesis. Histol Histopathol 25:795-806

45. Paradis R, Lazar N, Antinozzi P et al (2013) Nov/Ccn3, a novel transcriptional target of FoxO1, impairs pancreatic $\beta$-cell function. PLoS One 8:1-8
46. Chowdhury S, Wang X, Srikant CB et al (2014) IGF-I stimulates CCN5/WISP2 gene expression in pancreatic $\beta$-cells, which promotes cell proliferation and survival against streptozotocin. Endocrinology 155:1629-1642

47. Aly H, Rohatgi N, Marshall CA et al (2013) A novel strategy to increase the proliferative potential of adult human $\beta$-cells while maintaining their differentiated phenotype. PLoS One 8:e66131

48. Figeac F, Uzan B, Faro M et al (2010) Neonatal growth and regeneration of -cells are regulated by the Wnt/ -catenin signaling in normal and diabetic rats. AJP Endocrinol Metab 298:E245-E256

49. Mussmann R, Geese M, Harder F et al (2007) Inhibition of GSK3 promotes replication and survival of pancreatic beta cells. J Biol Chem 282:12030-12037 